\title{
CALABI QUASIMORPHISMS FOR MONOTONE COADJOINT ORBITS
}

\author{
ALEXANDER CAVIEDES CASTRO
}

\begin{abstract}
We show the existence of Calabi quasimorphisms on the universal covering $\overparen{\operatorname{Ham}}\left(\mathcal{O}_{\lambda}, \omega_{\lambda}\right)$ of the group of Hamiltonian diffeomorphisms of a monotone coadjoint orbit $\mathcal{O}_{\lambda}$ of a compact Lie group. We show that this result follows from positivity results of Gromov-Witten invariants and the fact that the quantum product of Schubert classes can never be zero.
\end{abstract}

\section{INTRODUCTION}

A quasimorphism of a group $G$ is a function $r: G \rightarrow \mathbb{R}$ for which exists a constant $C \geq 0$ such that

$$
\left|r\left(g_{1} g_{2}\right)-r\left(g_{1}\right)-r\left(g_{2}\right)\right| \leq C \quad \text { for every } g_{1}, g_{2} \in G \text {. }
$$

A quasimorphism $r$ is homogeneous if $r\left(g^{n}\right)=n r(g)$ for all $g \in G$ and $n \in \mathbb{Z}$.

A result of Banyaga [3] states that the universal covering $\widetilde{\operatorname{Ham}}(M, \omega)$ of the group of Hamiltonian diffeomorphisms of a closed symplectic manifold $(M, \omega)$ is perfect, i.e, it coincides with its commutator group. As a consequence $\widetilde{\operatorname{Ham}}(M, \omega)$ does not admit non-trivial homomorphisms to $\mathbb{R}$ when $M$ is closed.

Even under these circumstances, we still may be able to construct non-trivial quasimorphisms on $\widetilde{\operatorname{Ham}}(M, \omega)$ when $M$ is closed. Entov and Polterovich have constructed non-trivial quasimorphisms on $\widetilde{\operatorname{Ham}}(M, \omega)$ that in addition satisfy the Calabi condition with techniques coming from Floer and quantum homology [9], [10], [11], [12]. More precisely, Entov and Polterovich showed that if the quantum homology algebra $Q H_{*}(M)$ of a closed spherically monotone symplectic manifold $(M, \omega)$ contains a field as a direct summand, then $\widetilde{\operatorname{Ham}}(M, \omega)$ admits a homogeneous Calabi quasimorphism. In addition, Entov and Polterovich show that these Calabi quasimorphisms have potential applications in several areas of symplectic topology such as Hofer's geometry, $C^{0}$-symplectic topology and Lagrangian intersection theory (see e.g. Polterovich and Rosen's book [26]).

The quantum homology algebra $Q H_{*}(M)$ contains a field as a direct summand when $Q H_{*}(M)$ is for instance semisimple, i.e., $Q H_{*}(M)$ decomposes into direct sum of fields. Examples of symplectic manifolds with semi-simple quantum homology are projective spaces (see e.g. Entov and Polterovich [9]), complex grassmannian manifolds, the smooth complex quadric $\left\{z_{0}^{2}+z_{1}^{2}+\cdots+z_{n}^{2}=0\right\} \subset$ $\mathbb{C P}^{n}$ (see e.g. Abrams [2]), toric Fano 2-folds (see e.g. Ostrover and Tyomkin 
[25], Entov and Polterovich [11]), and generic symplectic toric manifolds (see e.g. Fukaya, Oh, Ohta, Ohno [13], Ostrover and Tyomkin [25]).

The quantum homology algebra of a symplectic manifold is endowed with a Frobenius structure. Abrams showed that a finite dimensional Frobenius algebra is semisimple if and only if its Euler class is invertible. Abrams used this criterion to show that (any specialization of) the quantum homology algebra of complex grassmannian manifolds is semisimple [2].

In this paper we focus our attention on coadjoint orbits $\mathcal{O}_{\lambda}$ of compact Lie groups. Coadjoint orbits of any Lie group are endowed with a symplectic form $\omega_{\lambda}$ known as the Kostant-Kirillov-Souriau form. The quantum cohomology algebra of a coadjoint orbit is not always semisimple. Chaput, Manivel and Perrin showed that the quantum homology algebra of the Grassmannian manifold of isotropic 2-planes in the six dimensional complex space $\mathbb{C}^{6}$ is not semisimple. They also showed that (any specialization of) the quantum homology algebra of (co)minuscule homogeneous spaces is semisimple [7].

Inspired by Abrams' result, in this paper we show that a finite dimensional Frobenius algebra over a field contains a field as a direct sum if and only if its Euler class is not nilpotent. We use this criterion to show that the quantum homology algebra of a coadjoint orbit of a compact Lie group always contains a field as a direct sum, even when it is not necessarily semisimple, and thus symplectic monotone coadjoint orbits of compact Lie groups admit Calabi quasimorphisms.

We can express the quantum Euler class of the quantum algebra of a coadjoint orbit $\mathcal{O}_{\lambda}$ of a compact Lie group in terms of the Schubert basis $\left\{\sigma_{u}\right\}$ of its homology group $H_{*}\left(\mathcal{O}_{\lambda}\right)$ as

$$
e_{q}=\sum_{u} \sigma_{u} * \check{\sigma}_{u}
$$

where in the last expression $*$ denotes the quantum product defined on $Q H_{*}\left(\mathcal{O}_{\lambda}\right)$ and $\left\{\check{\sigma}_{v}\right\}$ the Schubert basis dual to $\left\{\sigma_{u}\right\}$.

The no nilpotency of the the quantum Euler class $e_{q}=\sum \sigma_{u} * \check{\sigma}_{u}$ is a consequence of the following two results: the first, the positiviy of genus zero Gromov-Witten invariants whose constrains are Schubert classes (see e.g. Fulton and Pandharipande [14]); and the second, the fact that the quantum product of two Schubert classes does not vanish (Fulton and Woodward [15]).

In the last part of this paper, we estimate from above the Hofer-Zenhder capacity of a coadjoint orbit of a compact Lie group. We use an inequality due to G. Lu [21] where an upper bound for the Hofer-Zehnder capacity of a symplectic manifold is estimated in terms of its Gromov-Witten invariants. G. Lu's inequality [21] together with Fulton and Woodward's results [15] allow us to express an upper bound for the Hofer-Zehnder capacity of a coadjoint orbit in terms of its GKM-graph. We show that this inequality is sharp for regular coadjoint orbits of the unitary group $U(n)$. 


\section{ACKNOWLEDGMENTS}

I would like to thank Leonid Polterovich whose question gave the impulse to write this paper. I also would like to thank Yaron Ostrover for useful discussions. This research is supported by the Israel Science Foundation grants 178/13 and $1380 / 13$.

\section{Frobenius Algebras and Euler Class}

In this section we review briefly the defition of a Frobenius algebra and its Euler class. We show that a Frobenius algebra contains a field factor in its direct sum decomposition if and only if its Euler class is not nilpotent. Most of the material presented in this section is adapted from Abrams [1], [2].

Let $K$ be a field of characteristic 0 and let $(A, \cdot)$ be a finite dimensional commutative algebra over $K$ with unity. A Frobenius structure of $A$ is a linear form $f: A \rightarrow K$ such that the bilinear form

$$
\begin{aligned}
\eta: A \times A & \rightarrow k \\
(a, b) & \mapsto f(a \cdot b)
\end{aligned}
$$

is nondegenerete. The pair $(A, f)$ is called a Frobenius algebra.

Let $\left\{e_{i}\right\}$ be a basis for $A$ and $\left\{e_{j}^{*}\right\}$ be the corresponding dual basis relative to $\eta$, i.e., the basis such that

$$
\eta\left(e_{i}, e_{j}^{*}\right)=f\left(e_{i} \cdot e_{j}^{*}\right)=\delta_{i j},
$$

for all $i$ and $j$. The Euler class of $(A, f)$ is the element

$$
e_{A, f}:=\sum_{i} e_{i} \cdot e_{i}^{*}
$$

The Euler class $e_{A, f}$ is well defined and independent of the choice of basis Abrams [1. Proposition 5]. Note that $e_{A, f}$ is nonzero because

$$
f\left(e_{A, f}\right)=(A: K) \in K,
$$

and $K$ has characteristic 0 .

Given two Frobenius algebras $(A, f)$ and $(B, g)$, their direct sum is the pair $(A \oplus B, f \oplus g)$ where $A \oplus B$ denotes the orthogonal direct sum of algebras whose product is defined

$$
\left(a_{1}, b_{1}\right) \cdot\left(a_{2}, b_{2}\right):=\left(a_{1} \cdot b_{1}, a_{2} \cdot b_{2}\right),
$$

and $f \oplus g$ acts by

$$
\begin{aligned}
f \oplus g: A \oplus B & \rightarrow K \\
a \oplus b & \mapsto f(a)+g(b)
\end{aligned}
$$

If $A$ is an arbitrary algebra with direct sum decomposition of algebras $A=$ $\bigoplus_{i} A_{i}$, then $(A, f)$ is a Frobenius algebra for some $f$ if and only if for every $i$ there is a $f_{i}$ such that $\left(A_{i}, f_{i}\right)$ is a Frobenius algebra and $(A, f)=\bigoplus_{i}\left(A_{i}, f_{i}\right)$ 
Abrams [1]. Moreover, the Euler class $e_{A, f}$ respects direct sum decomposition of Frobenius algebras, i.e.,

$$
e_{A, f}=\bigoplus_{i} e_{A_{i}, f_{i}}
$$

We say that an algebra is indecomposable if it can not be written as a direct sum of two nonzero submodules. The Krull-Schmidt Theorem states that every finite dimensional algebra is a direct sum of indecomposable algebras. This decomposition is unique up to reordering of the summands (see e.g. Curtis and Reiner [8, Theorem 14.5]).

For a Frobenius algebra $(A, f)$ we denote its ideal of nilpotents by $\mathcal{N}(A)$. If the Frobenius algebra $(A, f)$ is indecomposable then the ideal of nilpotents $\mathcal{N}(A)$ consists of all non-units of $A$, and the annihilator of $\mathcal{N}(A)$ is the principal ideal generated by the Euler class $e_{A, f}$

$$
\operatorname{ann}(\mathcal{N}(A))=A \cdot e_{A, f}=\left\{a \cdot e_{A, f}: a \in A\right\}
$$

(Abrams 2, Proposition 3.3]). If $A$ is indecomposable and $\mathcal{N}(A)=0$, then $A$ contains only units and 0 , so $A$ is just a field extension of $K$, and in particular the Euler class $e_{A, f}$ is a unit. On the other hand, if $A$ is indecomposable and contains a nonzero nilpotent element $u$, then $u$ is a zero divisor of $e_{A, f}$ and $e_{A, f}$ is not a unit, and as a consequence the Euler class $e_{A, f}$ is nilpotent.

Theorem 2.1. A Frobenius algebra $(A, f)$ contains a field factor in its direct sum decomposition if and only if its Euler class $e_{A, f}$ is not nilpotent.

Proof. If there exists a field extension $(\mathcal{F}, g)$ of $K$ such that $(A, f)=(\mathcal{F}, g) \oplus$ $\left(A^{\prime}, f^{\prime}\right)$ for some Frobenius subalgebra $\left(A^{\prime}, f^{\prime}\right) \subset(A, f)$, then

$$
e_{(A, f)}=e_{(\mathcal{F}, g)} \oplus e_{\left(A^{\prime}, f^{\prime}\right)}
$$

The Euler class $e_{(\mathcal{F}, g)}$ is a unit and hence the Euler class $e_{(A, f)}$ can not be nilpotent.

On the other hand, if we decompose the Frobenius algebra $(A, f)$ into a direct sum of indecomposable components

$$
(A, f)=\bigoplus_{i}\left(A_{i}, f_{i}\right)
$$

and none of them is a field, then the Euler classes $e_{\left(A_{i}, f_{i}\right)}$ of the indecomposable components $\left(A_{i}, f_{i}\right)$ are nilpotents, and the Euler class

$$
e_{(A, f)}=\bigoplus_{i} e_{\left(A_{i}, f_{i}\right)}
$$

is nilpotent.

Theorem 2.2. The characteristic element $e_{(A, f)}$ of a Frobenius algebra $(A, f)$ is nilpotent if and only if $e_{(A, f)}^{2}=e_{(A, f)} \cdot e_{(A, f)}=0$. In particular, a Frobenius algebra $(A, f)$ contains a field factor in its direct sum decomposition if and only if $e_{(A, f)}^{2} \neq 0$. 
Proof. For any $\nu \in A$ there is a multiplication operator

$$
\begin{array}{r}
M_{\nu}: A \rightarrow A \\
a \mapsto a \cdot \nu
\end{array}
$$

It is not hard to see that

$$
\operatorname{Trace}\left(M_{\nu}\right)=f\left(e_{(A, f)} \cdot \nu\right)=\eta\left(e_{(A, f)}, \nu\right)
$$

Now if the characteristic element $e_{(A, f)}$ is nilpotent, then for any $\mu \in A$, the element $\nu=e_{(A, f)} \cdot \mu$ is nilpotent, and the corresponding multiplication operator $M_{\nu}=M_{e_{(A, f)} \cdot \mu}$ on $A$ have vanishing trace, so that

$$
\operatorname{Trace}\left(M_{\nu}\right)=\eta\left(e_{(A, f)}, \nu\right)=\eta\left(e_{(A, f)}, e_{(A, f)} \cdot \mu\right)=\eta\left(e_{(A, f)}^{2}, \mu\right)=0
$$

But $\eta$ is nondegenerate, and thus $e_{(A, f)}^{2}=0$.

A Frobenius algebra $(A, f)$ is semisimple if it is a direct sum of fields. The previous theorem is in contrast with Abrams' criterion for semisimplicity of Frobenius algebras:

Theorem 2.3 (Abrams [2]). A Frobenius algebra $(A, f)$ is semisimple if and only its Euler class $e_{(A, f)}$ is a unit.

Example 2.4. Let $f_{1}, \cdots, f_{n} \in \mathbb{C}\left[x_{1}, \cdots, x_{n}\right]$ be such that the quotient ring

$$
A=\mathbb{C}\left[x_{1}, \cdots, x_{n}\right] /\left\langle f_{1}, \cdots, f_{n}\right\rangle,
$$

is finite dimensional. The Local Duality Theorem states that a Frobenius structure for $A$ is given by the linear functional

$$
\begin{gathered}
f: A \rightarrow \mathbb{C} \\
F \rightarrow \int_{\left|f_{1}\right|=\cdots=\left|f_{n}\right|=1} \frac{F\left(x_{1}, \ldots, x_{n}\right)}{\prod_{i=1}^{n} f_{i}\left(x_{1}, \ldots, x_{n}\right)} d x_{1} \ldots d x_{n}
\end{gathered}
$$

(see e.g. Griffiths and Harris [18. Chapter 5]). The Euler class of $(A, f)$ can be written as

$$
e_{A, f}=u \cdot \operatorname{det}\left(\frac{\partial f_{i}}{\partial x_{j}}\right) \bmod \left\langle f_{1}, \cdots, f_{n}\right\rangle
$$

for some unit $u$ of $A$ (Abrams [1. Proposition 6.3]).

Let $Z=\left\{\lambda \in \mathbb{C}^{n}: f_{1}(\lambda)=\ldots=f_{n}(\lambda)=0\right\}$. The Euler class $e_{(A, f)}$ is a unit in $A$ if and only if $\operatorname{det}\left(\frac{\partial f_{i}}{\partial x_{j}}\right)(\lambda) \neq 0$ for all $\lambda \in Z$. Likewise, the Euler class $e_{(A, f)}$ is nilpotent in $A$ if and only if $\operatorname{det}\left(\frac{\partial f_{i}}{\partial x_{j}}\right)(\lambda)=0$ for all $\lambda \in Z$. So in conclusion,

(1) The Frobenius algebra $(A, f)$ is semisimple if and only if the multiplicity of any zero $\lambda \in Z$ is one.

(2) The Frobenius algebra $(A, f)$ contains a field factor in its direct sum decomposition if and only if there exists a zero $\lambda \in Z$ whose multiplicity is one. 


\section{Quantum Homology}

In this section we recall the definition of the quantum homology of a monotone spherically symplectic manifold. A standard reference of this material is McDuff and Salamon's book [23].

Let $\left(M^{2 n}, \omega\right)$ be a closed symplectic manifold. The symplectic manifold $\left(M^{2 n}, \omega\right)$ is spherically monotone if there exists a real constant $\kappa>0$ such that for all $A \in \pi_{2}(M)$

$$
c_{1}\left(T_{M}\right)(A)=\kappa \omega(A) .
$$

Here $c_{1}\left(T_{M}\right)$ denotes the first Chern class of the bundle $(T M, J)$, where $J$ is any almost complex structure compatible with $\omega$.

Let $\bar{\pi}_{2}(M)=\pi_{2}(M) / \sim$, where $A \sim B$ if and only if $\omega(A)=\omega(B)$. If we assume that $\omega$ does not vanish on $\pi_{2}(M)$, the group $\bar{\pi}_{2}(M)$ is an infinite cyclic group, and it has a generator $S$ so $\omega(S)>0$. The integer number $N:=$ $c_{1}\left(T_{M}\right)(S)$ is called the minimal Chern number of the monotone symplectic manifold $(M, \omega)$.

Let $A, B, C \in H_{*}(M, \mathbb{Z})$ be three homology classes. Let $J$ be a regular almost structure compatible with the symplectic form $\omega$. We denote by $\operatorname{GW}_{k}(A, B, C)$ the Gromov-Witten invariant that, roughly speaking, counts the number of $J$ holomorphic maps $\mathbb{C P}^{1} \rightarrow M$ representing the class $k S \in \bar{\pi}_{2}(M)$ and passing through generic representatives of the homology classes $A, B$ and $C$. The Gromov-Witten invariant $\mathrm{GW}_{k}(A, B, C)$ is zero unless

$$
\operatorname{deg} A+\operatorname{deg} B+\operatorname{deg} C=4 n-2 k N
$$

Let $\Lambda$ be the field of formal Laurent series whose principal part is a finite sum

$$
\Lambda:=\mathbb{C}[q]]=\left\{\begin{array}{c}
\sum_{k \in \mathbb{Z}} z_{k} q^{k}: z_{k} \in \mathbb{C}, \text { and there exists } \\
l \in \mathbb{Z} \text { such that } z_{k}=0 \text { for all } k \leq l
\end{array}\right\}
$$

As a vector space over $\mathbb{C}$ the quantum homology algebra of $(M, \omega)$ is defined to be

$$
Q H_{*}(M):=H_{*}(M) \otimes_{\mathbb{C}} \Lambda
$$

The quantum multiplication $*$ on $Q H_{*}(M)$ is defined as follows: for $A, B \in$ $H_{*}(M)$ and $k \in \mathbb{Z}_{\geq 1}$ define $(A * B)_{k} \in H_{*}(M)$ as the unique class such that

$$
(A * B)_{k} \circ C=\mathrm{GW}_{k}(A, B, C)
$$

for all $C \in H_{*}(M)$. Here o stands for the ordinary intersection index in homology. Now for any $A, B \in H_{*}(M)$ set

$$
A * B=A \cap B+\sum_{k \in \mathbb{Z}_{\geq 1}}(A * B)_{k} \otimes q^{k} \in Q H_{*}(M)
$$

By $\Lambda$-linearity, we can extend the quantum product to the whole $Q H_{*}(M)$. We define a grading on $Q H_{*}(M)$ so

$$
\operatorname{deg}\left(A \otimes q^{k}\right):=\operatorname{deg} A-2 k N
$$


for any $A \in H_{*}(M)$, and hence $\operatorname{deg}(a * b)=\operatorname{deg}(a)+\operatorname{deg}(b)-2 n$ for any $a, b \in Q H_{*}(M)$.

The quantum product defined on $Q H_{*}(M)$ is skew-commutative, associative and with unity equal to the fundamental class $[M]$. The even part $Q H_{\mathrm{ev}}(M):=$ $H_{\mathrm{ev}}(M) \otimes_{\mathbb{C}} \Lambda$ is a commutative subalgebra of $Q H_{*}(M)$.

The algebra $Q H_{\mathrm{ev}}(M)$ is a Frobenius algebra over the Field $\Lambda$ with Frobenius algebra structure $f$ that associates to a quantum homology class its coefficient at the fundamental class of a point $[\mathrm{pt}] \in H_{0}(M)$

$$
\begin{aligned}
f: Q H_{\mathrm{ev}}(M) & \rightarrow \Lambda \\
\sum_{A \in H_{*}(M)} A \otimes P_{A}(q) & \mapsto P_{[\mathrm{pt}]}
\end{aligned}
$$

The pairing

$$
\begin{aligned}
\eta: Q H_{\mathrm{ev}}(M) & \times Q H_{\mathrm{ev}}(M) \rightarrow \Lambda \\
(a, b) & \mapsto f(a * b)
\end{aligned}
$$

is non-degenerate and $\eta(a * b, c)=\eta(a, b * c)$ for all $a, b, c \in Q H_{\mathrm{ev}}(M)$.

\section{Calabi quasimorphisms and Quantum Homology}

In this section we remind the reader the relationship between quantum homology and Calabi quasimorphisms. The material presented in this section is mostly based on Entov and Polterovich's papers [9], [10], [11], [12].

Let $\left(M^{2 n}, \omega\right)$ be a closed connected symplectic manifold and $I \subset \mathbb{R}$ be an interval containing 0 . Given a smooth Hamiltonian function $H: I \times M \rightarrow \mathbb{R}$, set $H_{t}:=H(t, \cdot)$. We can associate the time dependent vector field $X_{H_{t}}$ defined by

$$
\iota_{X_{H_{t}}} \omega=d H_{t}
$$

We denote by $\phi_{H}^{t}$ the flow generated by $X_{H_{t}}$. A Hamiltonian $H(x, t)$ of the closed symplectic manifold $(M, \omega)$ is normalized if

$$
\int_{M} H_{t} \omega=0 \text { for all } t \in I
$$

The group of Hamiltonian diffeomorphisms are the time maps of Hamiltonian flows generated by normalized Hamiltonians

$$
\operatorname{Ham}(M, \omega):=\left\{\phi_{H}^{t}: H \text { (normalized) Hamiltonian }\right\}
$$

Let $\widetilde{\operatorname{Ham}}(M, \omega)$ be the universal covering of $\operatorname{Ham}(M, \omega)$

$$
\begin{array}{r}
\widetilde{\operatorname{Ham}}(M, \omega)=\left\{(\phi,[\alpha]): \phi \in \operatorname{Ham}(M, \omega), \alpha=\left\{\alpha_{t}\right\}_{t \in[0,1]}\right. \text { is a smooth } \\
\text { path of Hamiltonian diffeomorphisms with } \left.\alpha_{0}=I, \alpha_{1}=\phi\right\},
\end{array}
$$

here $[\alpha]$ stands for the homotopy class of $\alpha$ with fixed endpoints.

For a non-empty open subset $U$ of $M$, we denote by $\widetilde{\operatorname{Ham}}_{U}(M, \omega)$ the subgroup of $\widetilde{\operatorname{Ham}}(M, \omega)$ consisting of all elements that can be represented by a path 
$\left\{\phi_{H}^{t}\right\}_{t \in[0,1]}$ starting at the identity and generated by a Hamiltonian function $H_{t}$ supported in $U$ for all $t$. Consider the map

$$
\begin{aligned}
\mathrm{Cal}_{U} & : \widetilde{\operatorname{Ham}}_{U}(M, \omega) \rightarrow \mathbb{R} \\
\phi & \mapsto \int_{0}^{1} \int_{M} H_{t} \omega^{n}
\end{aligned}
$$

This map is well defined, i.e., it is independent of the choice of the Hamiltonian functions generating $\phi$. It is a group homomorphism called the Calabi homomorphism (see e.g. Polterovich and Rosen [26. Chapter 4]).

A non-empty subset $U$ of $M$ is called Hamiltonian displeceable if there exists a Hamiltonian diffeomorphism $\phi \in \operatorname{Ham}(M, \omega)$ such that $\phi(U) \cap \operatorname{Closure}(U)=\emptyset$.

A quasimorphism $\mu: \widetilde{\operatorname{Ham}}(M, \omega)$ is called a Calabi quasimorphism if it satisfies the following two properties:

(1) The map $\mu: \widetilde{\operatorname{Ham}}(M, \omega) \rightarrow \mathbb{R}$ coincides with the Calabi homomorphism $\mathrm{Cal}_{U}: \widetilde{\operatorname{Ham}}_{U}(M, \omega) \rightarrow \mathbb{R}$ on any open and Hamiltonian displaceable set $U$.

(2) For normalized Hamiltonians $F, G: M \times I \rightarrow \mathbb{R}$

$$
\int \min _{M}\left(F_{t}-G_{t}\right) d t \leq \frac{\mu\left(\phi_{G}\right)-\mu\left(\phi_{F}\right)}{\operatorname{Vol}(M, \omega)} \leq \int \max _{M}\left(F_{t}-G_{t}\right) d t
$$

Entov and Polterovich in [9] have constructed quasimorphisms on $\widetilde{\operatorname{Ham}}(M, \omega)$ in terms of spectral invariants. These invariants are given by a map

$$
c: Q H_{\mathrm{ev}}(M) \times \widetilde{\operatorname{Ham}}(M, \omega) \rightarrow \mathbb{R}
$$

We refer the reader to Entov and Polterovich [9], Oh [17], Polterovich and Rosen [26], Schwarz [27], Usher [28], etc. for more details about spectral invariants and their properties.

Theorem 4.1 (Entov and Polterovich [9]). Let $(M, \omega)$ be a closed monotone symplectic manifold. Assume that the quantum homology algebra $Q H_{\mathrm{ev}}(M)$ splits as an algebra as $\mathcal{F} \oplus \mathcal{R}$, where $\mathcal{F}$ is a field. Let $e$ be the unit of $\mathcal{F}$ and

$$
\begin{aligned}
c_{e}: \widetilde{\operatorname{Ham}}(M, \omega) & \rightarrow \mathbb{R} \\
\phi & \mapsto c(e, \phi)
\end{aligned}
$$

be the spectral invariant associated to $e$. Then, the function $c_{e}: \widetilde{\operatorname{Ham}}(M, \omega) \rightarrow$ $\mathbb{R}$ is a quasimorphism, and the homogenization $\mu: \widetilde{\operatorname{Ham}}(M, \omega) \rightarrow \mathbb{R}$ of $c_{e}$ given by

$$
\mu(\phi)=\lim _{m \rightarrow \infty} \frac{c_{e}\left(\phi^{m}\right)}{m}
$$

is a homogeneous Calabi quasimorphism. 


\section{Geometry of COADjoint orbits}

In this section we recall some general statements about the geometry of coadjoint orbits. Most of the material shown here can be found in the classical literature such as Kirillov [20] for the geometry of coadjoint orbits and Gelfand, Gelfand and Bernstein [4] for the geometry of Schubert varieties.

Let $G$ be a compact Lie group, $\mathfrak{g}$ be its Lie algebra and $\mathfrak{g}^{*}$ be the dual of $\mathfrak{g}$. Let $(\cdot, \cdot)$ denote an Ad-invariant inner product defined on $\mathfrak{g}$. We identify the Lie algebra $\mathfrak{g}$ and its dual $\mathfrak{g}^{*}$ via this inner product. Let $\lambda \in \mathfrak{g}^{*}$ and $\mathcal{O}_{\lambda} \subset \mathfrak{g}^{*}$ be the coadjoint orbit passing through $\lambda$. Let $\omega_{\lambda}$ be the Kostant-Kirillov-Souriau form defined on $\mathcal{O}_{\lambda}$ by

$$
\omega_{\lambda}(\hat{X}, \hat{Y})=\langle\lambda,[X, Y]\rangle \quad X, Y \in \mathfrak{g}
$$

where $\hat{X}, \hat{Y}$ are the vector fields on $\mathfrak{g}^{*}$ generated by the coadjoint action of $G$. The form $\omega_{\lambda}$ is closed and non-degenerate thus defining a symplectic structure on $\mathcal{O}_{\lambda}$.

We denote by $G_{\mathbb{C}}$ the complexification of the Lie group $G$. Let $P \subset G_{\mathbb{C}}$ be a parabolic subgroup of $G_{\mathbb{C}}$ such that $\mathcal{O}_{\lambda} \cong G_{\mathbb{C}} / P$. The quotient of complex Lie groups $G_{\mathbb{C}} / P$ allows us to endow $\mathcal{O}_{\lambda}$ with a complex structure $J$ compatible with $\omega_{\lambda}$ so the triple $\left(\mathcal{O}_{\lambda}, \omega_{\lambda}, J\right)$ is a Kähler manifold. The almost complex structure $J$ is regular in the sense of McDuff and Salamon 23, Proposition 7.4.3].

Let $T \subset G$ be a maximal torus and let $B \subset G_{\mathbb{C}}$ be a Borel subgroup with $T_{\mathbb{C}} \subset B \subset P$, where $T_{\mathbb{C}}$ denotes the complexification of the maximal torus $T \subset G$. Let $R \subset \mathfrak{t}^{*}$ be the root system of $T$ in $G$. Let $R^{+} \subset R$ be a system of positive roots with simple roots $S \subset R^{+}$. Let $W=N_{G}(T) / T$ be the Weyl group of $G$. For every root $\alpha \in R$, let $s_{\alpha} \in W$ be the reflection associated to it. Recall that the length $l(w)$ of $w \in W$ is defined as the minimum number of simple reflections $s_{\alpha} \in W, \alpha \in S$, whose product is $w$. For the parabolic subgroup $P \subset G_{\mathbb{C}}$, let $W_{P}=N_{P}(T) / T$ be the Weyl group of $P$ and $S_{P} \subset S$ be the subset of simple roots whose corresponding reflections are in $W_{P}$.

Let $w_{0}$ be the longest element in $W$ and let $B^{o p}:=w_{0} B w_{0} \subset G_{\mathbb{C}}$ be the Borel subgroup opposite to $B$. For $w \in W / W_{P}$, let $X(w):=\overline{B w P / P} \subset$ $G_{\mathbb{C}} / P$ and $Y(w):=\overline{B^{o p} w P / P} \subset G_{\mathbb{C}} / P$ be the Schubert variety and the opposite Schubert variety associated with $w$, respectively. We denote by $\sigma_{w}$ and $\check{\sigma}_{w}$ the fundamental classes in the homology group $H_{*}\left(G_{\mathbb{C}} / P, \mathbb{Z}\right)$ of $X(w)$ and $Y(w)$, respectively. Note that $\check{\sigma}_{w}=\sigma_{w_{o} w}$. For $w \in W / W_{P}$, we let $\check{w}:=$ $w_{0} w \in W / W_{P}$ so $\check{\sigma}_{w}=\sigma_{\check{w}}$. The set of Schubert classes $\left\{\sigma_{w}\right\}_{w \in W / W_{P}}$ forms a free $\mathbb{Z}$-basis of $H_{*}\left(G_{\mathbb{C}} / P, \mathbb{Z}\right)$, and the set of Schubert classes $\left\{\check{\sigma}_{w}\right\}_{w \in W / W_{P}}$ is 
the dual basis of $\left\{\sigma_{w}\right\}_{w \in W / W_{P}}$ with respect to the intersection pairing

$$
\begin{gathered}
H_{*}\left(G_{\mathbb{C}} / P, \mathbb{Z}\right) \otimes H_{*}\left(G_{\mathbb{C}} / P, \mathbb{Z}\right) \rightarrow \mathbb{Z} \\
(A, B) \mapsto \int_{G_{\mathbb{C}} / P} A \cap B
\end{gathered}
$$

Now we explain when a coadjoint orbit $\mathcal{O}_{\lambda}$ is symplectically monotone with respect to the Kostant-Kirillov-Souriau form $\omega_{\lambda}$. Each root $\alpha \in R$ has a coroot $\check{\alpha} \in \mathfrak{t}$. The coroot $\check{\alpha}$ is identified with $\frac{2 \alpha}{(\alpha, \alpha)} \in \mathfrak{t}$ via the invariant inner product $(\cdot, \cdot)$. The system of coroots is the set $\check{R}=\{\check{\alpha}: \alpha \in R\}$ and the simple coroots is the set $\check{S}=\{\check{\alpha}: \alpha \in S\}$. For $\alpha \in R$, let $\varpi_{\alpha} \in \mathfrak{t}^{*}$ denote the fundamental weight defined by

$$
\left\langle\varpi_{\alpha}, \check{\beta}\right\rangle=\delta_{\alpha, \beta},
$$

for any $\beta \in R$. Here $\langle\cdot, \cdot\rangle$ denotes the standard pairing $\langle\cdot, \cdot\rangle: \mathfrak{t} \otimes \mathfrak{t}^{*} \rightarrow \mathbb{R}$.

The cohomology group $H^{2}\left(G_{\mathbb{C}} / P ; \mathbb{Z}\right)$ can be identified with the span

$$
\mathbb{Z}\left\{\varpi_{\alpha}: \alpha \in S \backslash S_{P}\right\}
$$

and the homology group $H_{2}\left(G_{\mathbb{C}} / P ; \mathbb{Z}\right)$ with the quotient

$$
\mathbb{Z} \check{S} / \mathbb{Z} \check{S}_{P}
$$

For each $\alpha \in S \backslash S_{P}$, we identify the class $\sigma_{s_{\alpha}} \in H_{2}\left(G_{\mathbb{C}} / P, \mathbb{Z}\right)$ with $\check{\alpha}+$ $\mathbb{Z} \check{S}_{P} \in \mathbb{Z} \check{S} / \mathbb{Z} \check{S}_{P}$ and we identify its Poincaré dual $\operatorname{PD}\left(\sigma_{s_{\alpha}}\right) \in H^{2}\left(G_{\mathbb{C}} / P, \mathbb{Z}\right)$ $H^{2}\left(G_{\mathbb{C}} / P, \mathbb{Z}\right)$ with $\varpi_{\beta}$. When $\lambda \in \mathfrak{t}^{*}$, the cohomology class of the KostantKirillov-Souriau form $\left[\omega_{\lambda}\right] \in H^{2}\left(G_{\mathbb{C}} / P, \mathbb{Z}\right)$ is identified with $\lambda \in \mathbb{R}\left\{\varpi_{\alpha}\right.$ : $\left.\alpha \in S \backslash S_{P}\right\} \subset \mathbb{R}\left\{\varpi_{\alpha}: \alpha \in S\right\}=\mathfrak{t}^{*}$. The Poincaré pairing $H_{2}\left(G_{\mathbb{C}} / P, \mathbb{Z}\right) \otimes$ $H^{2}\left(G_{\mathbb{C}} / P, \mathbb{Z}\right) \rightarrow \mathbb{Z}$ is compatible with the standard pairing $\langle\cdot, \cdot\rangle: \mathfrak{t} \otimes \mathfrak{t}^{*} \rightarrow$ $\mathbb{R}$. For two degrees $c, d \in H_{2}\left(G_{\mathbb{C}} / P, \mathbb{Z}\right)$, we say that $c \leq d$, if $c-d=$ $\sum_{\alpha \in S \backslash S_{P}} b_{\alpha} \sigma_{s_{\alpha}}$ and $b_{\alpha} \in \mathbb{Z}_{\geq 0}$ for all $\alpha \in S \backslash S_{P}$.

Under these identifications,

$$
c_{1}\left(T_{G_{\mathbb{C}} / P}\right)=\sum_{\gamma \in R^{+} \backslash R_{P}^{+}} \gamma \in \mathbb{Z}\left\{\varpi_{\alpha}: \alpha \in S \backslash S_{P}\right\} \cong H^{2}\left(G_{\mathbb{C}} / P, \mathbb{Z}\right)
$$

A coadjoint orbit $\left(\mathcal{O}_{\lambda}, \omega_{\lambda}\right) \cong\left(G_{\mathbb{C}} / P, \omega_{\lambda}\right)$ is symplectically monotone if there exists a real constant $\kappa>0$ such that for all $\check{\alpha} \in \check{S} \backslash \check{S}_{P}$

$$
n_{\alpha}:=\int_{\sigma_{s_{\alpha}}} c_{1}\left(T_{G_{\mathbb{C}} / P}\right)=\left\langle\sum_{\gamma \in R^{+} \backslash R_{P}^{+}} \gamma, \check{\alpha}\right\rangle=\kappa\langle\lambda, \check{\alpha}\rangle=\kappa \omega_{\lambda}\left(\sigma_{s_{\alpha}}\right),
$$

or simply,

$$
\lambda=\frac{1}{\kappa} \sum_{\gamma \in R^{+} \backslash R_{P}^{+}} \gamma
$$

The minimal Chern number of the coadjoint orbit $\mathcal{O}_{\lambda}$ is given by

$$
N:=\underset{\alpha \in S \backslash S_{P}}{\operatorname{gcd}} n_{\alpha} .
$$




\section{Quantum hohomology of $G_{\mathbb{C}} / P$}

Now we define the quantum homology of $\mathcal{O}_{\lambda} \cong G_{\mathbb{C}} / P$. For a degree $d=$ $\sum_{\alpha \in S \backslash S_{P}} d_{\alpha} \sigma_{s_{\alpha}} \in H_{2}\left(G_{\mathbb{C}} / P, \mathbb{Z}\right)$ and $u, v, w \in W / W_{P}$, let $N_{u, v}^{w}(d)$ be the Gromov-Witten invariant $\mathrm{GW}_{d}\left(\sigma_{u}, \sigma_{v}, \check{\sigma}_{w}\right)$ that is equal to the number of morphisms $\mu: \mathbb{C P}^{1} \rightarrow X$ of degree $d$ such that for three given distinct points $p_{1}, p_{2}, p_{3} \in \mathbb{C P}^{1}$ and three general $g_{1}, g_{2}, g_{3} \in G_{\mathbb{C}}, \mu\left(p_{1}\right)$ is in $g_{1} \cdot X(u), \mu\left(p_{2}\right)$ is in $g_{2} \cdot X(v)$, and $\mu\left(p_{3}\right)$ is in $g_{3} \cdot Y(w)$, in particular the Gromov-Witten invariants $N_{u, v}^{w}(d)$ are nonnegative integer numbers (see e.g. Fulton and Pandharipande [14, Lemma 14]).

Let $\Lambda=\mathbb{C}[q]]$ be the field of Laurent series with complex coefficients and finite principal part. Recall that the quantum homology ring $Q H_{*}\left(G_{\mathbb{C}} / P\right)$ is as $\Lambda$-module the tensor product

$$
H_{*}\left(G_{\mathbb{C}} / P, \mathbb{Z}\right) \otimes_{\mathbb{C}} \Lambda
$$

so the Schuber classes $\left\{\sigma_{w}=\sigma_{w} \otimes 1\right\}_{w \in W / W_{P}}$ form a basis for $Q H_{*}\left(G_{\mathbb{C}} / P\right)$ over $\Lambda$. The quantum product $*$ is defined by

$$
\sigma_{u} * \sigma_{v}=\sigma_{u} \cap \sigma_{v}+\sum_{k \in \mathbb{Z}_{\geq 1}}\left(\sigma_{u} * \sigma_{v}\right)_{k} q^{k}
$$

where

$$
\left(\sigma_{u} * \sigma_{v}\right)_{k}=\sum_{w \in W / W_{P}}\left(\sum_{\sum_{\alpha} d_{\alpha} n_{\alpha}=k N} N_{u, v}^{w}(d)\right) \sigma_{w}
$$

Let

$$
f: Q H_{*}\left(G_{\mathbb{C}} / P\right)=H_{*}\left(G_{\mathbb{C}} / P\right) \otimes \Lambda \rightarrow \Lambda
$$

be the map defined by

$$
f\left(\sum_{u \in W / W_{P}} \sigma_{u} \otimes P_{u}(q)\right)=P_{e}(q)
$$

The bilinear map

$$
\begin{aligned}
\eta: Q H_{*}\left(G_{\mathbb{C}} / P\right) & \otimes Q H_{*}\left(G_{\mathbb{C}} / P\right) \rightarrow \Lambda \\
(u, v) & \mapsto f(u * v)
\end{aligned}
$$

defines a Frobenius algebra structure on $Q H_{*}\left(G_{\mathbb{C}} / P\right)$ over the field $\Lambda$.

Theorem 6.1. For $G_{\mathbb{C}} / P$, let $\sigma_{w} \in H_{*}\left(G_{\mathbb{C}} / P, \mathbb{Z}\right)$ be the Schubert class associated with $w \in W / W_{P}$. The quantum Euler class of the Frobenius algebra $\left(Q H_{*}\left(G_{\mathbb{C}} / P\right), f\right)$ is equal to

$$
e_{q}=\sum_{w \in W / W_{P}} \sigma_{w} * \check{\sigma}_{w} \in Q H_{*}\left(G_{\mathbb{C}} / P\right),
$$

where $\check{\sigma}_{w} \in H_{*}\left(G_{\mathbb{C}} / P, \mathbb{Z}\right)$ denotes the Schubert class opposite to $\sigma_{w}$. 
Proof. The Schubert classes $\left\{\sigma_{w}\right\}_{w \in W / W_{P}}$ form a basis for $Q H_{*}\left(G_{\mathbb{C}} / P\right)$ over $\Lambda$. We just have to check that the dual basis of $\sigma_{w}$ with respect to the Frobenius pairing $\eta$ is $\check{\sigma}_{w}$, or equivalently

$$
\eta\left(\sigma_{u}, \check{\sigma}_{v}\right)=f\left(\sigma_{u} * \check{\sigma}_{v}\right)=\delta_{u v}
$$

for all $u, v \in W / W_{P}$.

The basis $\left\{\check{\sigma}_{u}\right\}$ is dual to the basis $\left\{\sigma_{u}\right\}$ with respect to the intersection pairing defined on $H_{*}(G / P, \mathbb{Z})$, and as consequence

$$
f\left(\sigma_{u} \cap \check{\sigma}_{v}\right)=\delta_{u v}
$$

On the other hand, for a nonzero degree $d$, the Gromov-Witten invariant

$$
\mathrm{GW}_{d}\left(\sigma_{u}, \check{\sigma}_{v}, \check{\sigma}_{e}\right)=\mathrm{GW}_{d}\left(\sigma_{u}, \check{\sigma}_{v},[G / P]\right)=N_{u, \check{v}}^{e}(d)
$$

is zero (see e.g. McDuff and Salamon [23, Exercise 7.1.6]), therefore

$$
\eta\left(\sigma_{u}, \check{\sigma}_{v}\right)=f\left(\sigma_{u} * \check{\sigma}_{v}\right)=\sum_{\sum_{\beta} d_{\beta} n_{\beta}=k N} q^{k} N_{u, \check{v}}^{e}(d)=f\left(\sigma_{u} \cap \check{\sigma}_{v}\right)=\delta_{u v}
$$

and we are done.

Example 6.2 (Quantum Euler Class of $G(2,4)$ ). Let $G(2,4)$ be the complex Grassmannian manifold of 2-planes in $\mathbb{C}^{4}$. Shubert cycles in $H_{*}(G(2,4))$ are parametrized by elements in the quotient set $S_{4} /\left(S_{2} \times S_{2}\right)$. Elements in the quotient set $S_{4} /\left(S_{2} \times S_{2}\right)$ are in one to one correspondence with the partitions contained in the rectangle of size $2 \times 2$, i.e., the partitions

$$
\{0,1,(1,1), 2,(2,1),(2,2)\} \text {. }
$$

We will denote the corresponding Schubert cycles in the homology ring $H_{*}(G(2,4))$ by

$$
\left\{\sigma_{0}, \sigma_{1}, \sigma_{(1,1)}, \sigma_{2}, \sigma_{(2,1)}, \sigma_{(2,2)}\right\} .
$$

In our convention, for a partition $\lambda$

$$
\operatorname{dim}_{\mathbb{C}}\left(\sigma_{\lambda}\right)=\operatorname{dim}_{\mathbb{C}} G(2,4)-|\lambda|,
$$

where $|\lambda|$ denotes the size of the partition $\lambda$.

As a $\Lambda$-module

$$
Q H_{*}(G(2 ; 4)) \cong \operatorname{span}_{\Lambda}\left\langle\sigma_{0}, \sigma_{1}, \sigma_{(1,1)}, \sigma_{2}, \sigma_{(2,1)}, \sigma_{(2,2)}\right\rangle
$$

The special Schubert classes $\left\{\sigma_{0}, \sigma_{1}, \sigma_{2}\right\}$ generate $Q H_{*}(G(2 ; 4))$ as a ring. Giambelli's formula together with Pieri's formula determine all the multiplication of Schubert classes in the Grassmannian manifold (see e.g. Bertram [5]). We summarize the quantum multiplications of Schubert classes in the following table 


\begin{tabular}{|c|cccccc|}
\hline$*$ & 1 & $\sigma_{1}$ & $\sigma_{2}$ & $\sigma_{(1,1)}$ & $\sigma_{(2,1)}$ & $\sigma_{(2,2)}$ \\
\hline 1 & 1 & $\sigma_{1}$ & $\sigma_{2}$ & $\sigma_{(1,1)}$ & $\sigma_{(2,1)}$ & $\sigma_{(2,2)}$ \\
$\sigma_{1}$ & $\sigma_{1}$ & $\sigma_{2}+\sigma_{(1,1)}$ & $\sigma_{(2,1)}$ & $\sigma_{(2,1)}$ & $\sigma_{(2,2)}+q$ & $q \sigma_{1}$ \\
$\sigma_{2}$ & $\sigma_{2}$ & $\sigma_{(2,1)}$ & $\sigma_{(2,2)}$ & $q$ & $q \sigma_{1}$ & $q \sigma_{(1,1)}$ \\
$\sigma_{(1,1)}$ & $\sigma_{(1,1)}$ & $\sigma_{(2,1)}$ & $q$ & $\sigma_{(2,2)}$ & $q \sigma_{1}$ & $q \sigma_{2}$ \\
$\sigma_{(2,1)}$ & $\sigma_{(2,1)}$ & $\sigma_{(2,2)}+q$ & $q \sigma_{1}$ & $q \sigma_{1}$ & $q \sigma_{2}+q \sigma_{(1,1)}$ & $q \sigma_{(2,1)}$ \\
$\sigma_{(2,2)}$ & $\sigma_{(2,2)}$ & $q \sigma_{1}$ & $q \sigma_{(1,1)}$ & $q \sigma_{2}$ & $q \sigma_{(2,1)}$ & $q^{2}$ \\
\hline
\end{tabular}

The Quantum Euler class $e_{q}$ of $Q H_{*}(G(2,4))$ is equal to

$$
\begin{aligned}
e_{q} & =2 \sigma_{(2,2)} * 1+2 \sigma_{1} * \sigma_{(2,1)}+\sigma_{(1,1)} * \sigma_{(1,1)}+\sigma_{2} * \sigma_{2} \\
& =6 \sigma_{(2,2)}+2 q
\end{aligned}
$$

The Quantum Euler class $e_{q}$ has inverse $\frac{3}{16 q^{2}} \sigma_{(2,2)}-\frac{1}{16 q}$, and, in particular, the quantum cohomology ring $Q H_{*}(G(2,4))$ is semisimple. For arbitrary Grassmannian manifolds $G(k, n)$, Abrams has shown in [1] that the quantum homology ring $Q H_{*}(G(k, n))$ (after specialization of the quantum parameters) is semisimple by proving that the quantum Euler class of $Q H_{*}(G(k, n))$ is invertible.

The following is an example due to Chaput, Manivel and Perrin [7] of a homogeneous space $G_{\mathbb{C}} / P$ whose quantum cohomology ring $Q H^{*}\left(G_{\mathbb{C}} / P\right)$ is not semisimple.

Example 6.3 (Quantum Euler class of $I G(2,6)$ ). Let us endow $\mathbb{C}^{6}$ with a symplectic form $\Omega$. We denote by $S p(6, \mathbb{C})$ the symplectic group of invertible linear transformations on $\mathbb{C}^{6}$ that preserve $\Omega$.

We denote by $I G(2,6)$ the isotropic Grassmannian of isotropic 2-planes in $\mathbb{C}^{6}$. The dimension of $I G(2,6)$ is equal to 7 . Schubert cycles in $H_{*}(I G(2,6))$ are indexed by 1-strict partitions $\lambda$ (see e.g. Chaput, Manivel and Perrin [7]), and in $H_{*}(I G(2,6), \mathbb{Z})$ there are explicitly 12 of these classes

$$
\left\{\sigma_{0}, \sigma_{1}, \sigma_{2}, \sigma_{1,1}, \sigma_{3}, \sigma_{2,1}, \sigma_{4}, \sigma_{3,1}, \sigma_{4,1}, \sigma_{3,2}, \sigma_{4,2}, \sigma_{4,3}\right\}
$$

the degree of the Schubert class $\sigma_{\lambda}$ is equal to

$$
2\left(\operatorname{dim}_{\mathbb{C}}(I G(2,6))-\left(\lambda_{1}+\lambda_{2}\right)\right.
$$

In the figure below, we draw the Hasse diagram of the Schubert cycles in $H_{*}(I G(2,6), \mathbb{Z})$ ordered by inclusion of the corresponding Schubert varieties 


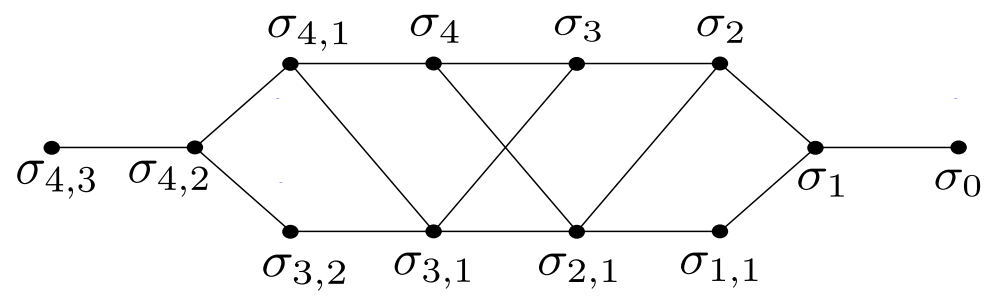

The following is the multiplication table of the Schubert classes $\sigma_{\lambda}$ with $\sigma_{1}, \sigma_{2}, \sigma_{3}$ in $Q H_{*}(I G(2,6))$ (see e.g. Chaput, Manivel and Perrin [7], and Galkin, Mellin and Smirnov [16])

\begin{tabular}{|c|cccc|}
\hline$*$ & & $\sigma_{1}$ & $\sigma_{2}$ & $\sigma_{3}$ \\
\hline$\sigma_{1}$ & $\sigma_{1}$ & $\sigma_{2}+\sigma_{1,1}$ & $\sigma_{3}+\sigma_{2,1}$ & $2 \sigma_{4}+\sigma_{3,1}$ \\
$\sigma_{2}$ & $\sigma_{2}$ & $\sigma_{3}+\sigma_{2,1}$ & $2\left(\sigma_{4}+\sigma_{3,1}\right)$ & $2 \sigma_{4,1}+\sigma_{3,2}+q \sigma_{0}$ \\
$\sigma_{1,1}$ & $\sigma^{2}-\sigma_{2}$ & $\sigma_{2,1}$ & $\sigma_{4}+\sigma_{3,1}$ & $\sigma_{4,1}+q \sigma_{0}$ \\
$\sigma_{3}$ & $\sigma_{3}$ & $2 \sigma_{4}+\sigma_{3,1}$ & $2 \sigma_{4,1}+\sigma_{3,2}+q \sigma_{0}$ & $2 \sigma_{4,2}+q \sigma_{1}$ \\
$\sigma_{2,1}$ & $\sigma_{1} * \sigma_{2}-\sigma_{3}$ & $\sigma_{4}+2 \sigma_{3,1}$ & $2 \sigma_{4,1}+\sigma_{3,2}+q \sigma_{0}$ & $\sigma_{4,2}+2 \sigma_{1} q$ \\
$\sigma_{4}$ & $\sigma_{1} * \sigma_{2,1}-2 \sigma_{3,1}$ & $\sigma_{4,1}+\sigma_{0} q$ & $\sigma_{4,2}+q \sigma_{1}$ & $\sigma_{4,3}+q \sigma_{2}$ \\
$\sigma_{3,1}$ & $-\frac{1}{3} \sigma_{1} *\left(\sigma_{3}-2 \sigma_{2,1}\right)$ & $\sigma_{4,1}+\sigma_{3,2}$ & $\sigma_{4,2}+q \sigma_{1}$ & $q\left(\sigma_{2}+\sigma_{1,1}\right)$ \\
$\sigma_{4,1}$ & $\sigma_{1} * \sigma_{4}-q$ & $\sigma_{4,2}+q \sigma_{1}$ & $\sigma_{4,3}+q\left(\sigma_{2}+\sigma_{1,1}\right)$ & $q\left(\sigma_{2,1}+\sigma_{3}\right)$ \\
$\sigma_{3,2}$ & $\sigma_{1} * \sigma_{3,1}-\sigma_{4,1}$ & $\sigma_{4,2}$ & $q \sigma_{2}$ & $q \sigma_{2,1}$ \\
$\sigma_{4,2}$ & $\sigma_{1} * \sigma_{4,1}-q \sigma_{1}$ & $\sigma_{4,3}+\sigma_{2} q$ & $q\left(\sigma_{3}+\sigma_{2,1}\right)$ & $q\left(2 \sigma_{3,1}+\sigma_{4}\right)$ \\
$\sigma_{4,3}$ & $\sigma_{1} * \sigma_{4,2}-q \sigma_{2}$ & $\sigma_{3} q$ & $q\left(\sigma_{4}+\sigma_{3,1}\right)$ & $q\left(\sigma_{4,1}+\sigma_{3,2}\right)$ \\
\hline
\end{tabular}

The quantum Euler class of $Q H_{*}(I G(2,6))$ equals to

$$
\begin{aligned}
e_{q} & =2\left(\sigma_{0} * \sigma_{4,3}+\sigma_{1} * \sigma_{4,2}+\sigma_{2} * \sigma_{4,1}+\sigma_{1,1} * \sigma_{3,2}+\sigma_{3} * \sigma_{4}+\sigma_{2,1} * \sigma_{3,1}\right) \\
& =2\left(6 \sigma_{4,3}+4 \sigma_{2} q+\sigma_{1,1} q\right)
\end{aligned}
$$

The Euler class $e_{q}$ is not invertible because it has zero divisors, for instance

$$
e *\left(\sigma_{4,3}-\sigma_{2} q+\sigma_{1,1} q\right)=0 .
$$

As a consequence, the quantum homology algebra $Q H_{*}(I G(2,6))$ is not semisimple.

In the next theorem, we show that the quantum homology algebra of any coadjoint orbit of a compact Lie group contains a field factor in its direct sum decomposition. 
Theorem 6.4. The quantum homology algebra $Q H_{*}\left(G_{\mathbb{C}} / P\right)$ contains a field factor in its direct sum decomposition.

Proof. By Theorem 2.1. the quantum cohomology algebra $Q H_{*}\left(G_{\mathbb{C}} / P\right)$ contains a field factor in its direct sum decomposition if and only if the square of the quantum Euler class

$$
e_{q}=\sum_{w \in W / W_{P}} \sigma_{w} * \check{\sigma}_{w}
$$

is nonzero. Note that the Euler class $e_{q}$ is of the form

$$
e_{q}=\operatorname{dim}_{\Lambda} Q H_{*}\left(G_{\mathbb{C}} / P\right)[\mathrm{pt}]+\sum_{\substack{u \in W / W_{P} \\ u \neq e}}\left(\sum_{k \in \mathbb{Z}_{\geq 0}} q^{k} c_{k, u}\right) \sigma_{u},
$$

where $c_{k, u}$ are nonnegative integer numbers. Thus $e_{q} * e_{q} \neq 0$, if for instance

$$
[\mathrm{pt}] *[\mathrm{pt}] \neq 0
$$

or if there exists a Schubert cycle $\sigma_{u}$ and some degree $c \in H_{2}\left(G_{\mathbb{C}} / P, \mathbb{Z}\right)$ so the Gromov-Witten invariant $\mathrm{GW}_{c}\left([\mathrm{pt}],[\mathrm{pt}], \sigma_{u}\right)$ is non-zero.

Let $w_{0}$ be the longest element in $W$. If we denote by $d$ the degree of any $T$-invariant curve joining the coset $P / P$ with the coset $w_{0} P / P$ in $G_{\mathbb{C}} / P$, by the Theorem 9.1 of Fulton and Woodward [15], there exists a degree $c \leq d$ and a Schubert class $\sigma_{u}$ so the Gromov-Witten invariant $\mathrm{GW}_{c}\left([\mathrm{pt}],[\mathrm{pt}], \sigma_{u}\right)$ is nonzero, and we are done.

As a consequence of the last result and Theorem 4.1, we obtain the following theorem

Theorem 6.5. Let $\left(\mathcal{O}_{\lambda}, \omega_{\lambda}\right)$ be a monotone coadjoint orbit of a compact Lie group. Then there exists a homogeneous Calabi quasimorphism on $\widetilde{\operatorname{Ham}}\left(\mathcal{O}_{\lambda}, \omega_{\lambda}\right)$.

Remark 6.6. In this paper we do not consider the question of whether the quasimorphism given by Theorem 6.5 descends to $\operatorname{Ham}\left(\mathcal{O}_{\lambda}, \omega\right)$. This is the same as showing that the Calabi quasimorphism vanishes on $\pi_{1}\left(\operatorname{Ham}\left(\mathcal{O}_{\lambda}, \omega_{\lambda}\right)\right)$. The Calabi quasimorphism restricted to $\pi_{1}\left(\operatorname{Ham}\left(\mathcal{O}_{\lambda}, \omega_{\lambda}\right)\right)$ can be written in terms of the Seidel representation (see e.g. Entov and Polterovich [9], McDuff [22]), but it is a difficult problem to compute the Seidel representation. However, partial results have been obtained in this direction. Entov and Polterovich showed that the Calabi quasimorpshims described in Theorem 6.5 descends when the coadjoint orbit $\mathcal{O}_{\lambda}$ is isomorphic to a projective space [9]. Also, Branson showed that the Calabi quasimorphism descends when the coadjoint orbit is isomorphic to the Grassmannian manifold $G(2,4)$ of 2-planes in $\mathbb{C}^{4}[6]$. Branson showed that the Seidel elements in $G(2,4)$ have "finite order" and the quantum homology algebra $Q H_{*}(G(2,4))$ has McDuff's "property D"; according to McDuff [22], when these two properties hold simultaneously, the Calabi quasimorphism descends. Unfortunately, Branson also showed that the projective spaces and the 
Grassmannian manifold $G(2,4)$ are the only Grassmannian manifolds satisfying property D. So even the problem of deciding when the Calabi quasimorphism of a Grassmannian manifold descends to $\operatorname{Ham}\left(\mathcal{O}_{\lambda}, \omega_{\lambda}\right)$ is still open.

\section{UPPER BOUND FOR THE HOFER-ZEHNDER CAPACITY}

In this section we point out that part of the argument given in the proof of Theorem 6.4 can be used to estimate from above the Hofer-Zenhder capacity of a coadjoint orbit of a compact Lie group.

Let $(M, \omega)$ be a closed symplectic manifold. The oscillation of a Hamiltonian function $H: M \rightarrow \mathbb{R}$ is

$$
\operatorname{osc} H:=\max H-\min H
$$

A Hamiltonian function $H: M \rightarrow \mathbb{R}$ is $\left(\pi_{1}\right)$ admissible if all (contractible) periodic orbits of the Hamiltonian vector field $X_{H}$ of period $<1$ are constant.

The Hofer-Zehnder capacity of a closed symplectic manifold $(M, \omega)$ may be expressed as

$$
\mathrm{c}_{\mathrm{HZ}}(M, \omega)=\sup \{\operatorname{osc} H: H: M \rightarrow \mathbb{R} \text { admissible }\},
$$

and the $\pi_{1}$-sensitive Hofer-Zehnder capacity of a closed manifold $(M, \omega)$ as

$$
\mathrm{c}_{\mathrm{HZ}}^{\circ}(M, \omega)=\sup \left\{\operatorname{osc} H: H: M \rightarrow \mathbb{R} \pi_{1} \text {-admissible }\right\}
$$

The following theorem is due to G. Lu 21]

Theorem 7.1. Suppose that $(M, \omega)$ admits a nonzero Gromov-Witten invariant of the form

$$
\mathrm{GW}_{d}([\mathrm{pt}],[\mathrm{pt}], A, \ldots, B)
$$

where $d \in H_{2}(M, \mathbb{Z}) /$ torsion and $A, \ldots, B$ are rational homology classes of even degree. Then

$$
\mathrm{c}_{\mathrm{HZ}}^{\circ}(M, \omega) \leq \omega(d),
$$

in particular

$$
\mathrm{c}_{\mathrm{HZ}}(M, \omega) \leq \omega(d) .
$$

Remark 7.2. In [28], Usher has provided a Hamiltonian Floer Theory proof of the last theorem. The tools that Usher developed in [28] were also used by him to show a criterion for $(M, \omega)$ to admit a Calabi quasimorphism. This criterion is among the same lines as Entov and Polterovich's Theorem 4.1.

For a coadjoint orbit $\mathcal{O}_{\lambda} \cong G_{\mathbb{C}} / P$, let $w_{0}$ be the longest element in $W$. If we denote by $d$ the degree of any $T$-invariant curve joining $P / P$ with $w_{0} P / P$, by the Theorem 9.1 of Fulton and Woodward [15], there exists a degree $c \leq d$ and a Schubert class $\sigma_{u}$ such that

$$
\mathrm{GW}_{c}\left([\mathrm{pt}],[\mathrm{pt}], \sigma_{u}\right) \neq 0 .
$$


A $T$-invariant curve of a coadjoint orbit $\mathcal{O}_{\lambda} \cong G_{\mathbb{C}} / P$ can be described in terms of its GKM-graph. The GKM graph of $\mathcal{O}_{\lambda}$ is the graph whose vertices are its $T$ fixed points and the edges are its irreducible $T$-invariant curves. The collection of points $w P / P$ for $w \in W$ is the set of all $T$-fixed points in $G_{\mathbb{C}} / P$. For each positive root $\alpha \in R^{+} \backslash R_{P}^{+}$there is a unique irreducible $T$-invariant curve $C_{\alpha}$ that contains $P / P$ and $s_{\alpha} P / P$. Any other irreducible $T$-invariant curve is of the form $w \cdot C_{\alpha}$ for some $w \in W$. Any $T$-invariant curve is a connected tree of irreducible $T$-invariant curves (see e.g. Fulton and Woodward [15]).

All these remarks and its consequences regarding Hofer-Zehnder are summarized in the following theorem

Theorem 7.3. Let $\mathcal{O}_{\lambda}$ be a coadjoint orbit of a compact Lie group $G$, and let $\omega_{\lambda}$ be the Kostant-Kirillov-Souriu form defined on it. Then

$$
\mathrm{c}_{\mathrm{HZ}}\left(\mathcal{O}_{\lambda}, \omega_{\lambda}\right) \leq \min _{A} \omega_{\lambda}(A),
$$

where the minimum is taken over all degrees $A \in H_{2}\left(\mathcal{O}_{\lambda}, \mathbb{Z}\right)$ of $T$-invariant curves joining $P / P$ with $w_{0} P / P$ in the GKM-graph.

The following Example illustrates the previous theorem for regular coadjoint orbits of $U(n)$.

Example 7.4. Let $\lambda=\left(\lambda_{1}>\lambda_{2}>\ldots>\lambda_{n-1}>\lambda_{n}\right) \in \mathbb{R}^{n}$, and

$$
\mathcal{H}_{\lambda}:=\left\{A \in \mathfrak{u}(n): A^{*}=-A, \operatorname{spectrum} A=-\lambda\right\}
$$

We can identify the set of skew-Hermitian matrices $\mathcal{H}_{\lambda}$ with a regular coadjoint orbit of $U(n)$ via the pairing

$$
\begin{aligned}
\mathfrak{u}(n) \times \mathfrak{u}(n) & \rightarrow \mathbb{R} \\
(X, Y) & \mapsto \operatorname{Trace}(X Y)
\end{aligned}
$$

Let $T=U(1)^{n} \subset U(n)$ be the maximal torus of diagonal matrices in $U(n)$. The corresponding system of roots associated with the torus $T$ is the set of vectors $\left\{e_{i}-e_{j}: i \neq j\right\} \subset \mathfrak{t} \cong \mathfrak{t}^{*}$.

Any $T$-fixed point of $\mathcal{H}_{\lambda}$ with respect to the conjugation action is a permutation of the diagonal matrix $i\left(\lambda_{1}, \ldots, \lambda_{n}\right)$. Two $T$-fixed points of $\mathcal{H}_{\lambda}$ are joined by one irreducible $T$-invariant curve if they differ by one transposition.

In order to find an upper for the Hofer Zehnder capacity of $\mathcal{H}_{\lambda}$, we want to find a chain of irreducible $T$-invariant curves joining the two $T$-fixed points $i\left(\lambda_{1}, \lambda_{2}, \ldots, \lambda_{n}\right)$ and $i\left(\lambda_{n}, \lambda_{n-1}, \ldots, \lambda_{1}\right)$. Let us consider the following chain of irreducible $T$-invariant curves

$$
\begin{aligned}
i\left(\lambda_{1}, \lambda_{2}, \ldots, \lambda_{n-1}, \lambda_{n}\right) & \stackrel{(1, n)}{\longrightarrow} i\left(\lambda_{n}, \lambda_{2}, \ldots, \lambda_{n-1}, \lambda_{1}\right) \\
& \stackrel{(2, n-1)}{\longrightarrow} \ldots \rightarrow i\left(\lambda_{n}, \lambda_{n-1}, \ldots, \lambda_{2}, \lambda_{1}\right),
\end{aligned}
$$


The degree of this chain is equal to $\sum_{k=1}^{\left[\frac{n-1}{2}\right]}\left[C_{(k, n-k)}\right]$ and its symplectic area is equal to

$$
\omega_{\lambda}\left(\sum_{k=1}^{\left[\frac{n-1}{2}\right]}\left[C_{(k, n-k)}\right]\right)=\sum_{k=1}^{\left[\frac{n-1}{2}\right]}\left\langle\lambda, \check{\alpha}_{k, n-k+1}\right\rangle=\frac{1}{2} \sum_{k=1}^{n}\left|\lambda_{k}-\lambda_{n-k+1}\right|,
$$

where $\alpha_{k, n-k+1}$ denotes the root $e_{k}-e_{n-k+1}$, and thus

$$
\mathrm{c}_{\mathrm{HZ}}\left(\mathcal{H}_{\lambda}, \omega_{\lambda}\right) \leq \frac{1}{2} \sum_{k=1}^{n}\left|\lambda_{k}-\lambda_{n-k+1}\right|
$$

Now we show that this inequality is sharp by constructing an admissible Hamiltonian function $H: \mathcal{H}_{\lambda} \rightarrow \mathbb{R}$ whose oscillation is equal to the right hand side of the last inequality.

The conjugation action of the torus $T$ on $\mathcal{H}_{\lambda}$ is Hamiltonian with moment map given by

$$
\begin{aligned}
\mu: \mathcal{H}_{\lambda} & \rightarrow \mathbb{R}^{n} \\
A=\left(a_{i j}\right) & \rightarrow \operatorname{diagonal}(-i A)=\left(a_{11}, \ldots, a_{n n}\right)
\end{aligned}
$$

The image of the moment map $\mu$ is the convex hull of all possible permutations of the vector $\left(\lambda_{1}, \ldots, \lambda_{n}\right) \in \mathbb{R}^{n}$ (see, e.g. Guillemin [19]).

For $t \in U(1)$ and $\left(m_{1}, \ldots, m_{n}\right) \in \mathbb{Z}^{n}$, we will use the convention that

$$
t^{\left(m_{1}, \ldots, m_{n}\right)}:=\left(t^{m_{1}}, \ldots, t^{m_{n}}\right) \in T=U(1)^{n} \subset U(n) .
$$

Let

$$
\beta=\sum_{k=1}^{\left[\frac{n-1}{2}\right]}\left(e_{k}-e_{n-k+1}\right)
$$

and $S=\left\{t^{\beta}=\left(t, t, \ldots, t^{-1}, t^{-1}\right): t \in S^{1}\right\} \subset T$. The action of the circle $S$ on $\mathcal{H}_{\lambda}$ is Hamiltonian with moment map given by

$$
\begin{aligned}
\tilde{\mu}: \mathcal{H}_{\lambda} & \rightarrow \mathbb{R} \\
A=\left(a_{i j}\right) & \mapsto(\mu(A), \beta)=a_{1,1}-a_{n, n}+a_{2,2}-a_{n-1, n-1}+\ldots
\end{aligned}
$$

The moment map image of $\tilde{\mu}$ is the interval

$$
\left[-\frac{1}{2} \sum_{k=1}^{n}\left|\lambda_{k}-\lambda_{n-k+1}\right|, \frac{1}{2} \sum_{k=1}^{n}\left|\lambda_{k}-\lambda_{n-k+1}\right|\right] \subset \mathbb{R}
$$

and thus the oscillation of $\tilde{\mu}$ is equal to $\sum_{k=1}^{n}\left|\lambda_{k}-\lambda_{n-k+1}\right|$. Unfortunately, the function $\tilde{\mu}$ is not admissible. This is because, under the action of $S$ on $\mathcal{H}_{\lambda}$, there are elements in $\mathcal{H}_{\lambda}$ with non-trivial finite stabilizers. All possible stabilizer subgroups of $S$ are either $\{1\}, \mathbb{Z}_{2}$ or $S$. When the stabilizer subgroup of a skew-Hermitian matrix in $\mathcal{H}_{\lambda}$ is $\mathbb{Z}_{2}$, the period of the orbit passing through the skew-Hermitian matrix is $1 / 2$. Otherwise, the skew-Hermitian matrix is either 
a $S$-fixed point or the period of the orbit passing through the skew-Hermitian matrix is 1 .

The Hamiltonian function $H=\frac{1}{2} \tilde{\mu}: \mathcal{H}_{\lambda} \rightarrow \mathbb{R}$ fixes this problem. The orbits of $H$ are either constant or their periods are either 1 or 2 . So, $H$ is admissible, and

$$
\operatorname{osc}(H)=\frac{1}{2} \sum_{k=1}^{n}\left|\lambda_{k}-\lambda_{n-k+1}\right| \leq c_{\mathrm{HZ}}\left(\mathcal{H}_{\lambda}, \omega_{\lambda}\right)
$$

In conclusion,

$$
\mathrm{c}_{\mathrm{HZ}}\left(\mathcal{H}_{\lambda}, \omega_{\lambda}\right)=\frac{1}{2} \sum_{k=1}^{n}\left|\lambda_{k}-\lambda_{n-k+1}\right|
$$

\section{BIBLIOGRAPHY}

[1] Lowell Abrams, Two-dimensional topological quantum field theories and frobenius algebras, J. Knot Theory Ramifications 5 (1996).

[2] - The quantum Euler class and the quantum cohomology of the Grassmannians., Isr. J. Math. 117 (2000), 335-352 (English).

[3] Augustin Banyaga, Sur la structure du groupe des difféomorphismes qui préservent une forme symplectique, Comment. Math. Helv. 53 (1978), no. 2, 174-227. MR490874 (80c:58005)

[4] I. N. Bernstein, I. M. Gelfand, and S. I. Gelfand, Schubert cells, and the cohomology of the spaces $G / P$, Uspehi Mat. Nauk 28 (1973), no. 3(171), 3-26. MR0429933 (55 \#2941)

[5] Aaron Bertram, Quantum Schubert calculus, Adv. Math. 128 (1997), no. 2, 289-305. MR1454400 (98j:14067)

[6] Mark Branson, Symplectic manifolds with vanishing action-Maslov homomorphism, Algebr. Geom. Topol. 11 (2011), no. 2, 1077-1096. MR2792374 (2012d:53268)

[7] P. E. Chaput, L. Manivel, and N. Perrin, Quantum cohomology of minuscule homogeneous spaces iii. semi-simplicity and consequences, Canad. J. Math. 62 (2010), no. 6, 1246-1263.

[8] Charles W. Curtis and Irving Reiner, Representation theory of finite groups and associative algebras, Pure and Applied Mathematics, Vol. XI, Interscience Publishers, a division of John Wiley \& Sons, New York-London, 1962. MR0144979 (26 \#2519)

[9] Michael Entov and Leonid Polterovich, Calabi quasimorphism and quantum homology, Int. Math. Res. Not. 30 (2003), 1635-1676. MR1979584 (2004e:53131)

[10] Q Quasi-states and symplectic intersections, Comment. Math. Helv. 81 (2006), no. 1, 75-99. MR2208798 (2006k:53149)

[11] Symplectic quasi-states and semi-simplicity of quantum homology, Toric topology, 2008, pp. 47-70. MR2428348 (2010e:53151)

[12] _ Rigid subsets of symplectic manifolds, Compos. Math. 145 (2009), no. 3, 773826. MR2507748 (2011a:53174)

[13] Kenji Fukaya, Yong-Geun Oh, Hiroshi Ohta, and Kaoru Ono, Lagrangian Floer theory on compact toric manifolds. I, Duke Math. J. 151 (2010), no. 1, 23-174. MR2573826 (2011d:53220)

[14] W. Fulton and R. Pandharipande, Notes on stable maps and quantum cohomology, Algebraic geometry-Santa Cruz 1995, 1997, pp. 45-96. MR1492534 (98m:14025)

[15] W. Fulton and C. Woodward, On the quantum product of Schubert classes, J. Algebraic Geom. 13 (2004), no. 4, 641-661. MR2072765 (2005d:14078)

[16] S. Galkin, A. Mellit, and M. Smirnov, Dubrovin's conjecture for $I G(2,6)$, ArXiv e-prints (May 2014), available at 1405.3857 
[17] Yong geun Oh, Construction of spectral invariants of hamiltonian diffeomorphisms on general symplectic manifolds, 2004.

[18] Phillip Griffiths and Joseph Harris, Principles of algebraic geometry, Wiley-Interscience [John Wiley \& Sons], New York, 1978. Pure and Applied Mathematics. MR507725 (80b:14001)

[19] Victor Guillemin, Moment maps and combinatorial invariants of Hamiltonian $T^{n}$ spaces, Progress in Mathematics, vol. 122, Birkhäuser Boston Inc., Boston, MA, 1994. MR1301331 (96e:58064)

[20] A. A. Kirillov, Lectures on the orbit method, Graduate Studies in Mathematics, vol. 64, American Mathematical Society, Providence, RI, 2004. MR2069175 (2005c:22001)

[21] Guangcun Lu, Gromov-Witten invariants and pseudo symplectic capacities, Israel J. Math. 156 (2006), 1-63. MR2282367 (2009d:53133)

[22] Dusa McDuff, Monodromy in Hamiltonian Floer theory, Comment. Math. Helv. 85 (2010), no. 1, 95-133. MR2563682 (2011d:53222)

[23] Dusa McDuff and Dietmar Salamon, J-holomorphic curves and symplectic topology, Second, American Mathematical Society Colloquium Publications, vol. 52, American Mathematical Society, Providence, RI, 2012. MR2954391

[24] Yong-Geun Oh, Normalization of the Hamiltonian and the action spectrum, J. Korean Math. Soc. 42 (2005), no. 1, 65-83. MR2106281 (2005i:53117)

[25] Yaron Ostrover and Ilya Tyomkin, On the quantum homology algebra of toric Fano manifolds, Selecta Math. (N.S.) 15 (2009), no. 1, 121-149. MR2511201 (2010f:53158)

[26] Leonid Polterovich and Daniel Rosen, Function theory on symplectic manifolds, CRM Monograph Series, vol. 34, American Mathematical Society, Providence, RI, 2014. MR3241729

[27] Matthias Schwarz, On the action spectrum for closed symplectically aspherical manifolds, Pacific J. Math. 193 (2000), no. 2, 419-461. MR1755825 (2001c:53113)

[28] Michael Usher, Spectral numbers in Floer theories, Compos. Math. 144 (2008), no. 6, 1581-1592. MR2474322 (2010b:53156)

E-mail address: alexanderc1@post.tau.ac.il 